\title{
Wellens' syndrome: a case report with atypical features
}

\author{
Giulia Ghizzoni, Edoardo Sciatti, Enrico Vizzardi, Ivano Bonadei, Davide Fabbricatore, Marco Metra \\ Cardiology Unit, Department of Medical and Surgical Specialties, Radiological Sciences and Public Health, University \\ of Brescia; Cardio-Thoracic Department, ASST Spedali Civili, Brescia, Italy
}

\begin{abstract}
Wellens' syndrome is a pattern of electrocardiographic T-wave changes seen in the precordial leads in patients with unstable angina. Two different patterns have been described. Type A presents with biphasic T-waves in V2-V3 and type B presents with symmetrical deep T-wave inversions in V2-V3. The etiology is related to a critical stenosis in the proximal left anterior descending artery (LAD). We describe a patient who presented at our emergency department with intermittent chest pain and showing Wellens' pattern T-wave inversions in the precordial leads of the electrocardiogram. She was found to have $95 \%$ stenosis in mid LAD.
\end{abstract}

\section{Introduction}

Wellens' syndrome is a condition in which typical electrocardiogram (ECG) changes, either a biphasic T-wave or deep symmetrical T-wave inversions in the precordial leads, occur due to a critical left anterior descending artery (LAD) stenosis. The ECG does not demonstrate any pathological Q wave or ST segment variation. Such ECG changes usually occur during the pain-free period. It is crucial to identify this pattern and manage the patient by invasive revascularization at the earliest opportunity. If left untreated or medically managed, the patient may develop an extensive myocardial infarction or sudden death. The knowledge of what lies behind

Correspondence: Dr. Edoardo Sciatti, Cardiology Unit, University of Brescia and ASST Spedali Civili, Brescia, Italy.

Tel.: +39.030.3995536 - Fax: +39.030.3995013.

E-mail: edoardo.sciatti@gmail.com

Key words: Wellens' syndrome; unstable angina; electrocardiogram; T-wave.

Conflict of interest: the authors declare no conflict of interest.

Received for publication: 14 May 2019.

Accepted for publication: 4 September 2019.

${ }^{\circ}$ Copyright: the Author(s), 2019

Licensee PAGEPress, Italy

Monaldi Archives for Chest Disease 2019; 89:1093

doi: 10.4081/monaldi.2019.1093

This article is distributed under the terms of the Creative Commons Attribution Noncommercial License (by-nc 4.0) which permits any noncommercial use, distribution, and reproduction in any medium, provided the original author(s) and source are credited. such an ECG is also pivotal, as these patients are contraindicated from undergoing a stress test like treadmill test.

We will present a variant case of Wellens' syndrome.

\section{Case Report}

A 46-year-old female presented to our emergency department complaining multiple episodes of retrosternal chest pain at rest, each episode lasting for few minutes. The pain radiated to her back and left arm and was associated with slight breathlessness and chest tightness. She gave history of intermittent, exertional chest pain in the week before, which had resolved spontaneously. She was a smoker, hypertensive, overweight and with a positive family history of coronary artery disease.

At visit she was conscious, oriented and asymptomatic. Her heart rate was regular at $84 \mathrm{bpm}$; blood pressure was 180/110 $\mathrm{mmHg}$. The systemic examination was essentially normal. The routine laboratory tests were normal. The serum biomarkers of cardiac injury were negative: troponine I was $0.017 \mathrm{ng} / \mathrm{ml}$ (normal value $<0.045 \mathrm{ng} / \mathrm{ml}$ ). An ECG taken on admission when she was asymptomatic showed T-wave inversion in leads V2-V6 with no ST deviation, normal $\mathrm{R}$ wave progression in the precordial leads and no pathological Q waves (Figure 1). This pattern was thought to be suggestive of Wellens' syndrome indicating a critical LAD obstruction. The echocardiogram did not reveal any kinetic change of the left ventricle. A coronary computed tomography angiography (CTA) was performed showing a tight stenosis in the mid LAD artery due to the presence of a noncalcific coronary plaque with features of vulnerable lesion, extending longitudinally for $10 \mathrm{~mm}$.

The patient was admitted to our Cardiology Unit and she was administered dual antiplatelet therapy (acetylsalicylic acid $100 \mathrm{mg}$ od and ticagrelor $90 \mathrm{mg}$ bid), antihypertensive drugs and a highdose statin. Coronary angiography confirmed a $95 \%$ stenosis of the mid LAD (Figure 2A). Interventricular artery, left circumflex artery, and right coronary artery were patent. Percutaneous transluminal coronary angioplasty (PTCA) was performed and a drugeluting stent was placed in the LAD, after which the flow reverted back to normal (Figure 2B). The ECG taken after reperfusion therapy showed persistent deep T-wave inversions in V2-V6 (Figure 3). The patient was advised to continue standard medical therapy on discharge and concerning lifestyle changes, in particular she was counselled against the use of tobacco.

\section{Discussion}

Wellens' syndrome was first described by De Zwann et al. in a study group of 145 patients with unstable angina, 26 of them 
showing the ECG pattern of Wellens' syndrome [1]. Twelve patients out of this group who were not revascularized developed an extensive anterior wall infarction within few weeks after admission. Usually, two different patterns of precordial T-wave abnormality are seen in Wellens' syndrome: type A and type B. Type A has biphasic T-waves with initial positivity and terminal negativity (it was seen in about $25 \%$ of the cases). Type B has T-waves which are deep and symmetrically inverted (it was seen in about $75 \%$ of the cases). This syndrome was thought to represent a critical stenosis of the proximal LAD.

Later on, the same authors studied a larger sample: out of 1260 patients admitted to the hospital because of unstable angina, 180 had Wellens' ECG features and were all found to have a stenosis in the proximal LAD ranging from $50 \%$ to $100 \%$ [2].

Rhinehardt et al. described the following diagnostic criteria for Wellens' syndrome [3]: i) symmetric and deeply inverted T-waves in V2-3 (occasionally in leads V1, V4, V5 and V6) or biphasic T wave in leads V2 and V3; ii) isoelectric or minimally elevated $(<1$ $\mathrm{mm}$ ) ST-segment; iii) no precordial Q waves; iv) history of angina; v) ECG pattern present in the pain-free state; vi) normal or slightly elevated serum cardiac markers.

Throughout the years, many cases have been reported with

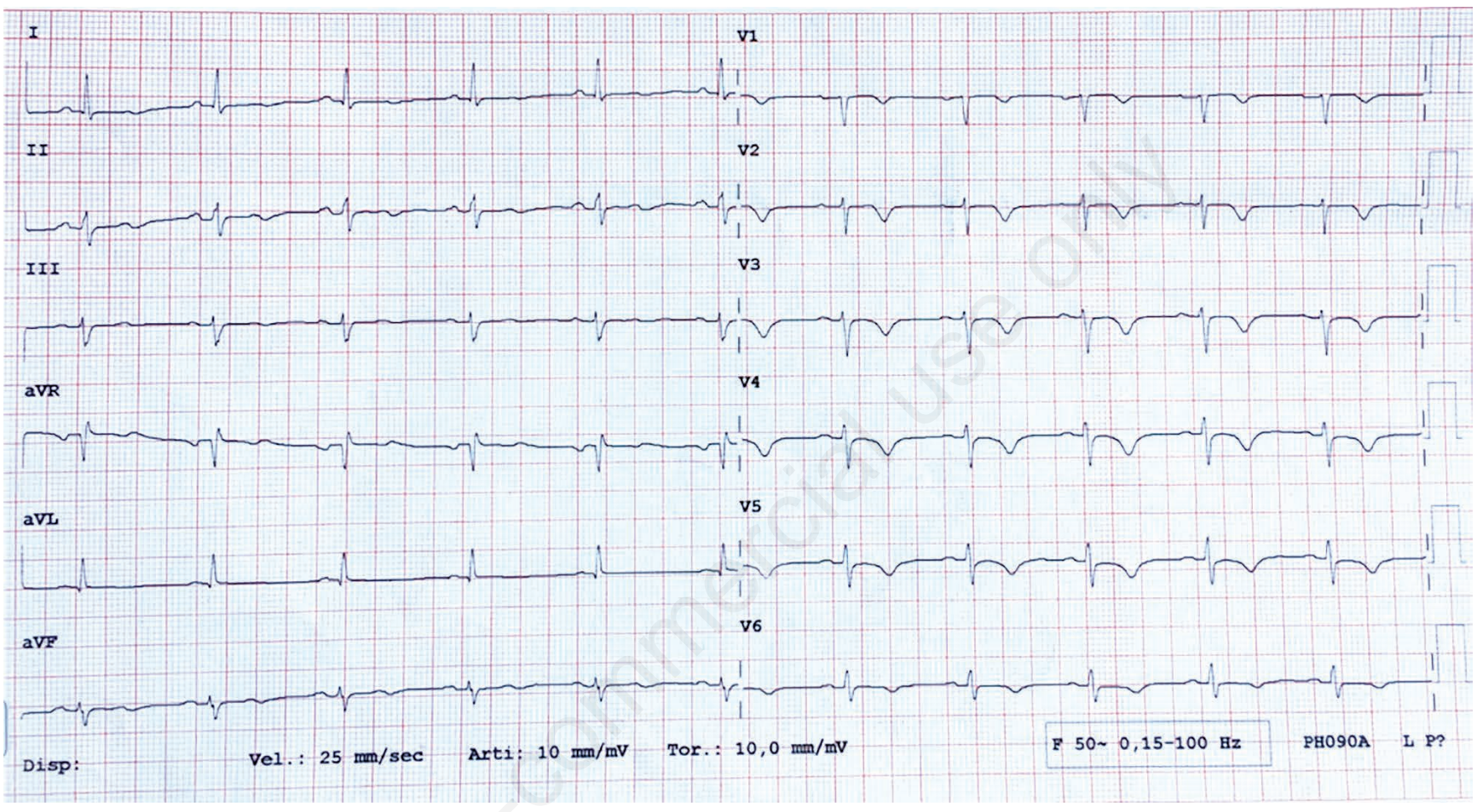

Figure 1. ECG at the Emergency Department.

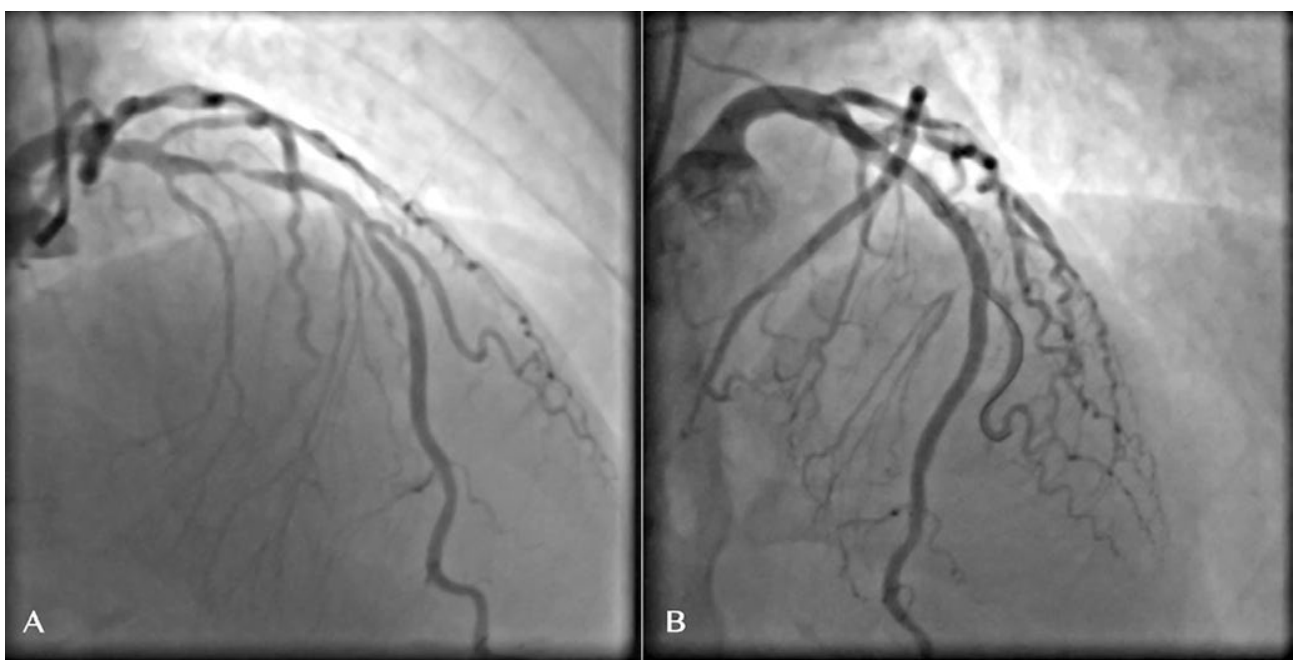

[page 6]
Figure 2. A) Coronary angiography showing mid left anterior descending artery stenosis. B) Drug-eluting stent treatment of the stenosis. 


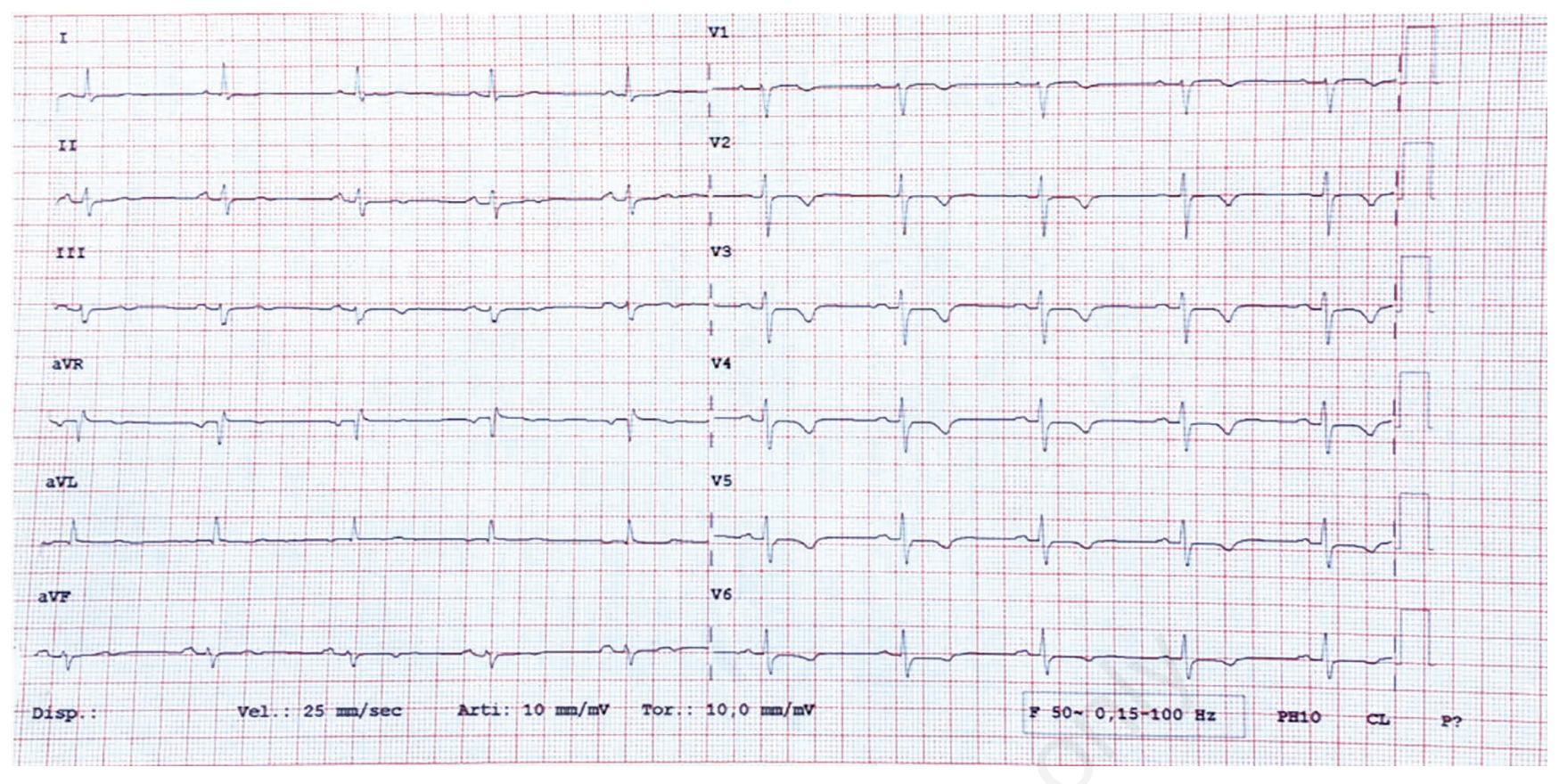

Figure 3. ECG after revascularization.

typical and atypical features of Wellens' syndrome. Kardesoglu et al. described a case where the ECG pattern changed from type B to type A during observation and in which the critical stenosis was in mid LAD [4]. Grautoff et al. presented a case of pre-existing left bundle branch block and chest pain. The ECG turned out to be consistent with type A Wellens' syndrome. The patient underwent cardiac catheterization showing a subtotal mid LAD stenosis [5]. Kyaw et al. reported an elderly woman with isolated throat pain and Wellens' pattern in the initial ECG, which prompted the proper workup and management. Subsequently, coronary angiogram revealed more than $90 \%$ occlusion of the proximal LAD artery and a drug-eluting stent was deployed [6]. Recently, Mufti et al. described a patient who was admitted for an elective surgery without a history of chest pain noted to have Wellens' pattern T-wave inversions on telemetry. She was found to have $60-70 \%$ stenosis in mid LAD [7].

The case we presented above highlights that variants in Wellens' syndrome exist. The coronary lesion was in mid LAD instead of the classical proximal part of LAD. Left main coronary artery, left circumflex artery, and right coronary artery had no significant stenosis.

In conclusion, Wellens' syndrome is a pattern of T-wave changes in the precordial leads that occurs in a subset of patients with unstable angina and meaning critical stenosis of the proximal LAD. It can have an atypical presentation, and our case highlights a patient with ECG characteristics of Wellens' syndrome but stenosis of mid LAD instead of proximal. Such patients may be a subgroup of Wellens' syndrome, but further debate will be necessary on this issue. More studies need to be carried on to fully understand the correlation between ECG changes and the different locations of coronary lesions. Early diagnosis and treatment of
Wellens' syndrome prevent the patient from developing myocardial infarction or sudden death. The knowledge of what lies behind such an ECG is also pivotal, as these patients are contraindicated from undergoing a stress test like treadmill test.

\section{References}

1. de Zwaan C, Bar FW, Wellens HJ. Characteristic electrocardiographic pattern indicating a critical stenosis high in left anterior descending coronary artery in patients admitted because of impending myocardial infarction. Am Heart J 1982;103:730-6.

2. de Zwaan C, Bar FW, Janssen JH, et al. Angiographic and clinical characteristics of patients with unstable angina showing an ECG pattern indicating critical narrowing of the proximal LAD coronary artery. Am Heart J 1989;117:657-65.

3. Rhinehardt J, Brady WJ, Perron AD, Mattu A. Electrocardiographic manifestations of Wellens' syndrome. Am J Emerg Med 2002;20:638-43.

4. Kardesoglu E, Celik T, Cebeci BS, et al. Wellens' syndrome: a case report. J Int Med Res 2003;31:585-90.

5. Grautoff S. Wellens' syndrome can indicate high-grade LAD stenosis in case of left bundle branch block. Herzschrittmacherther Elektrophysiol 2017;28:57-9.

6. Kyaw K, Latt H, Aung SSM, et al. Atypical presentation of acute coronary syndrome and importance of Wellens' syndrome. Am J Case Rep 2018;19:199-202.

7. Mufti M, Joea R, Sobnosky S, Longtine J. Wellens' Syndrome: An Atypical Presentation of an Already Silent Killer. J Med Cases 2018;9:173-6. 